\title{
Moving towards a technical specification for fluorescence excitation-emission mapping and absorbance analysis of colored dissolved organic matter
}

\author{
A. Gilmore ${ }^{1}$, R. Hurteaux ${ }^{2}$, S. FitzGerald ${ }^{3} \&$ A. Knowles ${ }^{3}$ \\ ${ }^{1}$ HORIBA Scientific, Edison, New Jersey, USA \\ ${ }^{2}$ HORIBA Jobin Yvon S.A.S., Chilly-Mazarin, France \\ ${ }^{3}$ HORIBA Jobin Yvon Ltd., Stanmore, UK
}

\begin{abstract}
Colored Dissolved Organic Matter (CDOM) measurements with fluorescence and absorbance are important for a variety of natural water sources. Uncertainties have been propagated regarding CDOM spectral data from different instruments, sampling conditions and analysis algorithms. Recent efforts have thus focused on standardizing sample preparation, spectroscopic evaluation, and mathematical analysis to minimize interlaboratory variation The practical significance of simultaneous acquisition of the corrected absorbance spectrum and fluorescence excitation-emission map is discussed as a means to provide the least ambiguity. Variation can be caused by different light-exposure history (especially UV) in separate instruments, dissolved-oxygen content from temperature changes, and oxidation kinetics. Concentration changes in CDOM can be associated with optical anomalies, including self-quenching and -absorption, which systematically alter the fluorescence spectrum. Monitoring the absorbance and fluorescence simultaneously avoids these sampling variations and facilitates spectral correction. The proposed method(s) described are discussed regarding their potential as the basis for an international technical specification of the instrument and sampling conditions for CDOM analysis and reporting.
\end{abstract}

Keywords: natural organic matter, colored dissolved organic matter, excitation-emission matrix, UV-VIS absorbance spectroscopy, inner-filter effect, primary inner-filter effect, secondary inner-filter effect, quinine sulfate unit, Rayleigh scattering, water Raman scattering. 


\section{Introduction}

Due to the increasing shortages of fresh, unpolluted drinking and irrigation water resources and pollution of the oceans globally, the analysis of colored dissolved organic matter (CDOM) is a key research area of international significance. Analysis of the often dilute and complex mixtures of CDOM components in water is made much easier using fluorescence technology, because it can yield parts-per-billion (ppb) sensitivity for many organic compounds and it is relatively inexpensive, simple to use, and nondestructive in nature compared to other techniques currently employed.

Fluorescent CDOM can comprise both natural and man-made components [1]. The natural components primarily consist of decomposition products of plant and animal materials, including humic and fulvic acids, proteins, and aromatic amino-acid constituents. Man-made fluorescent components can include petroleum products, fertilizers, pesticides, herbicides, sewage, pharmaceuticals, and - of increasing concern - toxic nanomaterials. CDOM influences natural bodies of water in several ways. Two of the primary effects are on the absorbance and light penetration-depth and on the oxygen demand (both biological and chemical), both of which are vital to sustaining life in water. CDOM is directly related to the oxygen demand, and hence viability, of natural water bodies because it consumes oxygen upon photodegradation, especially under the influence of UV light exposure.

The most common CDOM fluorescence-measurement technique is known as the excitation-emission map or EEM $[1,2]$. An EEM is acquired by scanning the excitation (absorbance) spectrum of the sample while simultaneously acquiring the fluorescence emission spectrum at each excitation wavelength coordinate. The EEM is important for CDOM analysis because, in contrast to a typical twodimensional absorbance scan of a water sample, the EEM exhibits a third dimension to provide both the absorbance and emission spectra of all fluorescent components. Thus an EEM strongly increases selectivity and potentially facilitates not only qualitative but quantitative analysis of all the samples' components.

One challenge in using fluorescence to analyze these samples is that, in most CDOM samples, the fluorescence spectral components can exhibit significant overlapping features. Current methodologies to separate, identify and quantify the components have focused on conventional non-linear least-squares spectral deconvolution as well as a variety of multivariate techniques [1-6]. The latter multivariate methods include, among others, Partial Least Squares (PLS), Principal Component Analysis (PCA), and the recently popularized Parallel Factor Analysis or PARAFAC method, which we focus on in this review.

The key issues surrounding analysis of the EEMs with PARAFAC are the importance of reproducible and accurate spectral and instrumental correction of the data $[1,2,6,7,9]$. A major required spectral-correction method discussed below involves the coordinated evaluation of the absorbance spectrum of the sample in order to correct for the optical processes known as "inner-filter effects" (IFEs) [10-13]. IFEs manifest in concentrated CDOM samples as both 
absorbance of the excitation beam (primary inner filter effect), and also sample fluoresence signals (secondary inner-filter effect), where the absorbance spectra and emission spectral regions of components overlap. IFE correction is significant in light of its role in referencing spectral libraries.

In most CDOM analyses, component identification is based on a public or privately-generated spectral library. Obviously, both intra- and interlaboratory comparisons must rely on traceable and reproducible spectral-correction procedures for the identifications to be validated [8]. This is of great concern given the diversity of instruments and conditions under which EEMs are reported in the literature for CDOM. Hence the final section of this article outlines our view of the future of fluorescent CDOM measurement for both research and analytical applications with regard to several prominent international efforts to standardize the EEM methodology.

\section{A focus on HORIBA's fluorescence instrumentation for water quality: the AquaLog ${ }^{\circledR}$}

When laboratory budgets and portability considerations are important, the benchtop AquaLog ${ }^{\circledR}$ is suitable for many CDOM laboratories because it exhibits the world's highest water-sensitivity rating for a benchtop fluorometer; it employs a thermoelectrically-cooled charge-coupled device (CCD) array detector for ultimate speed and sensitivity, is portable (can be operated directly on a ship), is fully corrected for the excitation source and instrument response, and also is equipped with the transmission detector needed for IFE correction.

The AquaLog ${ }^{\circledR}$ (fig. 1) is optimally configured for CDOM analysis using a xenon-arc light source to excite many UV-absorbing CDOM components of interest (1). The configuration shown also makes measurements of turbid and solid samples easier by virtue of an aberration-corrected double-grating excitation monochromator to remove stray light (2). Central to the AquaLog ${ }^{\circledR}$ is the sample compartment that is optically configured for simultaneous absorbance and fluorescence (90 degrees) data acquisition (3). The AquaLog ${ }^{\circledR}$ is equipped with a reference detector (4a) to monitor and ratiometrically correct both the excitation source's spectrum for the emission detector and the absorbance detector's signals. A transmission detector (4b) is attached to the AquaLog ${ }^{\circledR}$ 's sample compartment to record the sample's transmission/absorbance spectrum under the same spectral-bandpass and -resolution conditions as the fluorescence EEM data, which is required for accurate IFE corrections. Most important for the CDOM application, however, is that the AquaLog ${ }^{\circledR}$ is configured with an aberration-corrected $140 \mathrm{~mm}$ focal-length spectrograph with a thermoelectrically-cooled CCD detector (4c), which enables EEM collection at rates faster than one per minute. The $\mathrm{CCD}$ provides an unrivaled multichannel signal-to-noise advantage compared to scanning monochromator single-channeldetector-based systems. Speed is important because many CDOM studies involve hundreds of EEM samples. The system's control electronics are contained in the base (5). 


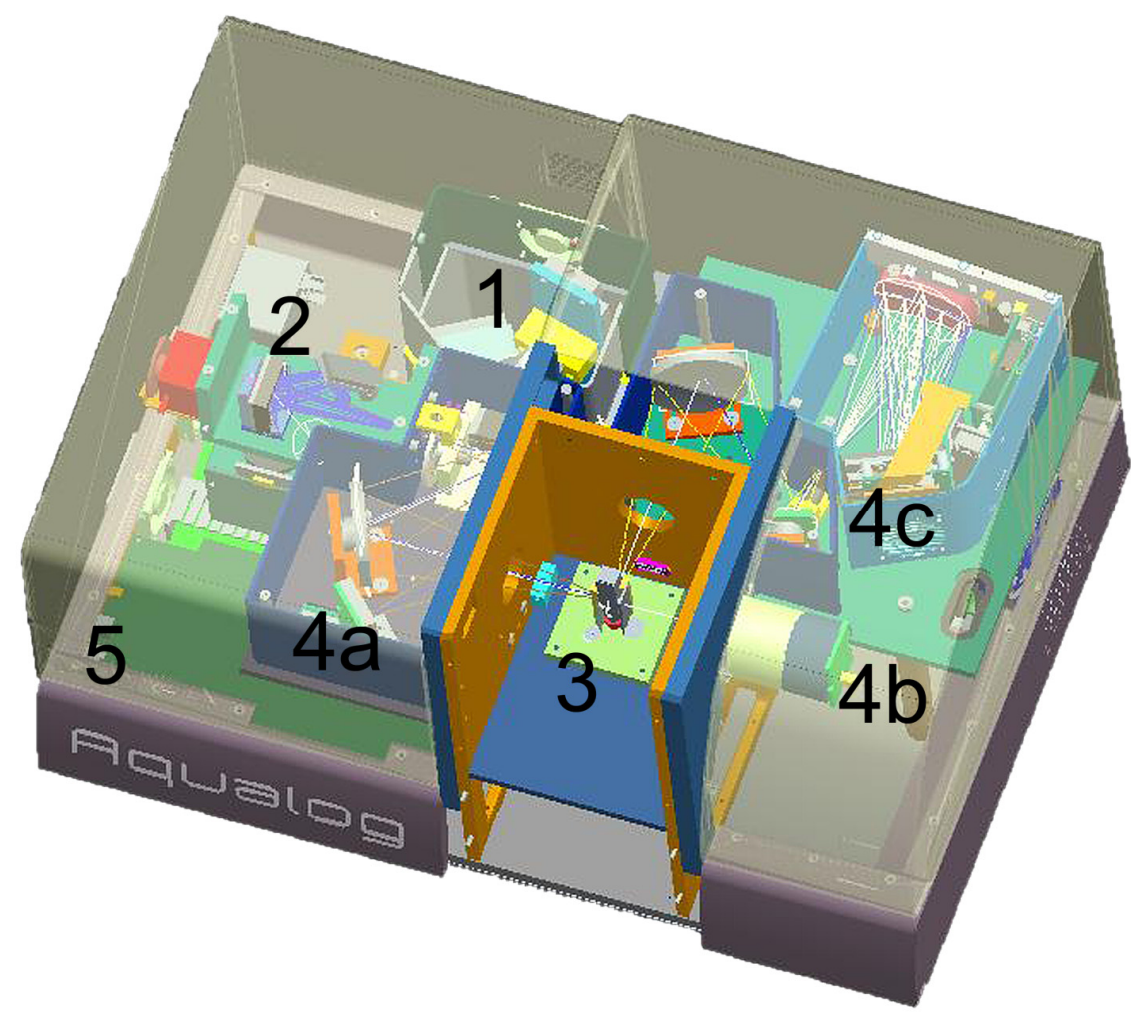

Figure 1: The optical layout for a bench-top AquaLog ${ }^{\circledR}$ configured for rapidly measuring excitation-emission maps of colored dissolved organic matter. Numerical labels designate major components of the unit, described in the paragraph above.

\section{An overview of the method of excitation-emission mapping with absorbance (IFE) analysis of CDOM}

A typical EEM (Fig. 2A) for a CDOM sample involves scanning the excitation wavelength from 240-500 $\mathrm{nm}$ and acquiring the emission spectra from 250-600 $\mathrm{nm}$. Spectral-bandpass and resolution conditions are now generally accepted to be $5 \mathrm{~nm}$ by the research community [8]. Because the excitation axis is scanned and the excitation source exhibits wavelength-dependent intensity features, the emission detector signal must be divided by the respective excitation-source intensity monitored using the reference $(R)$ detector at each excitation coordinate. Additionally both the $\mathrm{R}$ detector and emission $(S)$ detector signals require correction for the instrument's spectral responsivity, which conventionally includes subtracting the dark-current signal from each detector in 


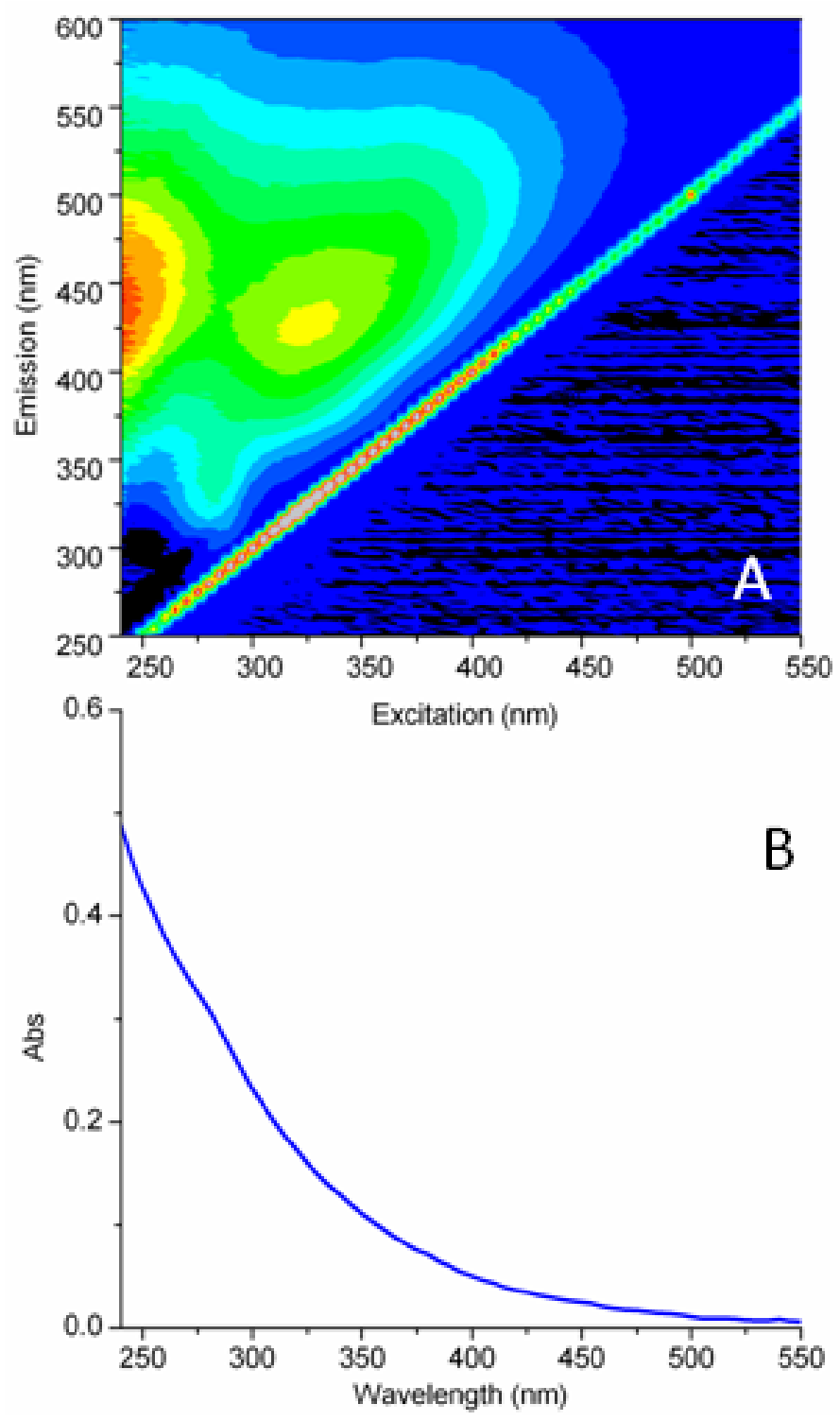

Figure 2: An EEM of the Pony Lake Fulvic Acid standard sample from the International Humic Substance Society (A). This map, and all maps shown below, were collected using $5 \mathrm{~nm}$ optical bandpass at 0.1 second integration time per emission spectrum. Panel B shows the absorbance spectrum of the sample shown in (A) measured under the same bandpass and integration time conditions. 
addition to multiplying the $R$ and $S$ signals by their respective excitation (Xcorrect) and emission (Mcorrect) spectral correction factors. Hence, the final EEM signal is recorded in our AquaLog ${ }^{\circledR}$ software using the general formula $S_{\mathrm{c}} / R_{\mathrm{c}}$ where $S_{\mathrm{c}}=(S-$ dark $) \times$ Mcorrect and $R_{\mathrm{c}}=(R-$ dark $) \times$ Xcorrect. In conjunction to the EEM fluorescence signal, the sample's absorbance spectral data (Figure 2B) can be collected in parallel by measuring the transmission $(A)$ detector signal formula $I=A_{\mathrm{c}} / R_{\mathrm{c}}$, where $A_{\mathrm{c}}=(A-$ dark $)$ and $R_{\mathrm{c}}=(R-$ dark $) \times$ Xcorrect. To measure the absorbance spectrum of the sample, one must also measure the $I_{\mathrm{o}}=\left(A_{\mathrm{c}} / R_{\mathrm{c}}\right)$ of a blank or reference sample to calculate Abs = $\log \left(I_{0} / I\right)$ as a function of wavelength. The conventional reference or blank sample is usually highly purified water $(\geq 18.2 \mathrm{M} \Omega,<2 \mathrm{ppb}$ total organic carbon). Note that the blank sample can serve several additional purposes as described below relative to correcting and processing the EEM data for qualitative and quantitative spectral analysis.

\section{EEM data processing: instrumental, spectral, and IFE correction with AquaLog ${ }^{\circledR}$ software}

Figure 3A shows for a blank sample of ultra-pure water that the current practice for EEMs involves measuring the excitation and emission scan ranges, which includes their overlap regions. These overlap regions manifest in intense signals from the monochromated excitation source in the emission detector's response; these lines are caused by both the first- (and second-) order Rayleigh-scattering features consistent with the well-known grating equation. Figure $3 \mathrm{~A}$ also shows another spectral feature, associated with the ultra-pure water sample, known as the water Raman scattering line. The Raman scattering line is related to the Rayleigh scattering line by a constant energy shift of $3382 \mathrm{~cm}^{-1}$. Most CDOM component libraries contain spectra for which the artifactual Rayleigh and waterRaman spectral features have been removed, and hence EEM data must be processed to remove these features systematically. Figure 3B shows the raw EEM for a CDOM sample, namely an aliquot of a standard sample known as the Pony Lake Fulvic Acid (PLFA) sample sourced from the International Humic Substance Society (IHSS). Here the main contours of the CDOM components are observed, along with the Rayleigh and Raman line scattering features. The AquaLog $^{\circledR}$ software package can remove both artifacts. Figure $3 \mathrm{C}$ shows the result of subtracting the blank EEM data in Fig. 3A from the CDOM sample data in Fig. 3B, which effectively removes the Raman scatter line. Figure 3D shows the results of applying the Rayleigh-masking algorithm, which nullifies the signal intensities for both the first- and second-order Rayleigh lines. As mentioned above, the water Raman signal of ultra-pure water is often used to normalize the signal intensities of the CDOM sample EEMs; similarly many researchers choose to normalize the CDOM EEMs based on a unit of fluorescence from quinine sulfate (QSU) $[1,6,12]$. Importantly, the AquaLog ${ }^{\circledR}$ EEM-processing software performs both the water Raman and QSU normalization. 


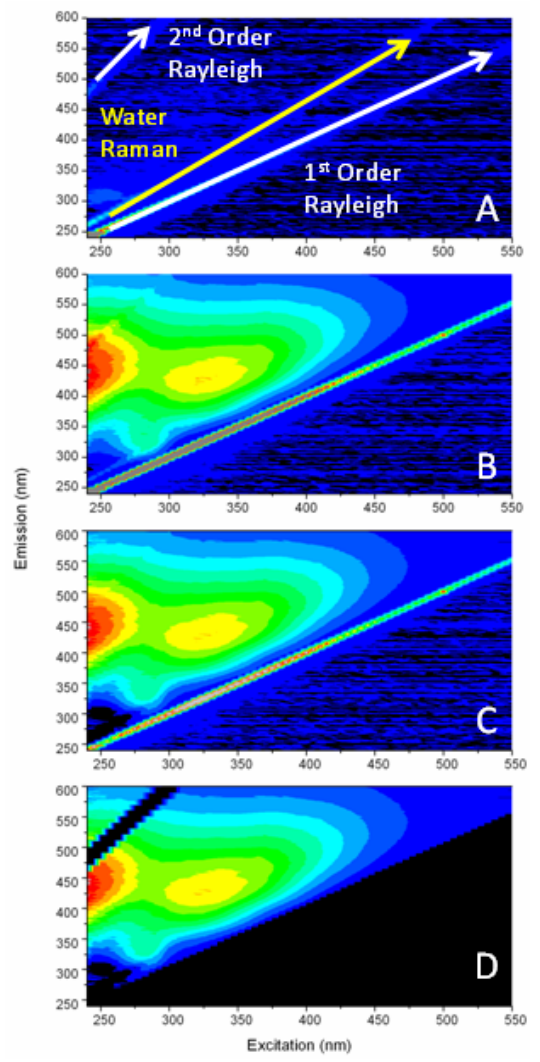

Figure 3: The fundamental instrument-correction operations for processing an EEM, including blank subtraction and Rayleigh-line nullification. The EEM of an ultra-pure water sample serving as the blank or reference sample showing the characteristic first- and second-order Rayleigh scattering lines (white) and water Raman scattering line in yellow (A). An uncorrected EEM of the Pony Lake Fulvic Acid standard sample (B). Panel C shows the result of subtracting the EEM data from the blank sample (A) from the sample in (B). Panel D shows the results of the algorithm that nullifies the first- and second-order Rayleigh lines from the map shown in $(\mathrm{C})$.

In addition to the fundamental water Raman and Rayleigh correction procedures, it is common practice to correct the EEM data for IFE using the parallel absorbance measurements from the sample and blank as described above for Figure 2. The IFE algorithms used in AquaLog ${ }^{\circledR}$ require measuring the absorbance spectrum of the sample covering the overlapping range of both the excitation and emission spectra to correct for both the primary and secondary IFE. The most common IFE algorithm used for conventional $1 \times 1 \mathrm{~cm}$ path- 
length cuvettes employs eqn. (1) applied to each excitation-emission wavelength coordinate of the EEM [11]:

$$
F_{\text {ideal }}=F_{\text {obs }}^{10} \frac{\mathrm{Abs}_{\mathrm{Ex}}+\mathrm{Abs} \mathrm{Em}^{2}}{2} .
$$

$F_{\text {ideal }}$ is the ideal fluorescence-signal spectrum expected in the absence of IFE, $F_{\text {obs }}$ is the observed fluorescence signal, and $\mathrm{Abs}_{\mathrm{Ex}}$ and $\mathrm{Abs}_{\mathrm{Em}}$ are the measured absorbance values at the respective excitation and emission wavelengthcoordinates of the EEM. A number of advanced algorithms described in the literature can also account for variations of the optical geometrical parameters of the cuvette path-length, beam- or slit-width, and positioning/shifting of the cuvette relative to the excitation and emission beam paths [10-13].

Figures $4 \mathrm{~A}$ and $\mathrm{B}$ show a comparison of EEMs collected using both a concentrated $\left(\mathrm{Abs}_{254 \mathrm{~nm}} \approx 0.8\right)$ PLFA sample to one diluted with ultra-pure water $\left(\mathrm{Abs}_{254 \mathrm{~nm}} \approx 0.2\right)$ in Figures $4 \mathrm{C}$ and $\mathrm{D}$. The absorbance value at $254 \mathrm{~nm}$ is an industry standard for evaluating the total CDOM concentration [8]. The EEMs on the left show the uncorrected ( $F_{\text {obs }}$ ) while the EEMs on the right show the IFE corrected $\left(F_{\text {ideal }}\right)$ for the corresponding samples. It is clear that for the uncorrected, higher-absorbing sample in Figure 4A that the spectral contours
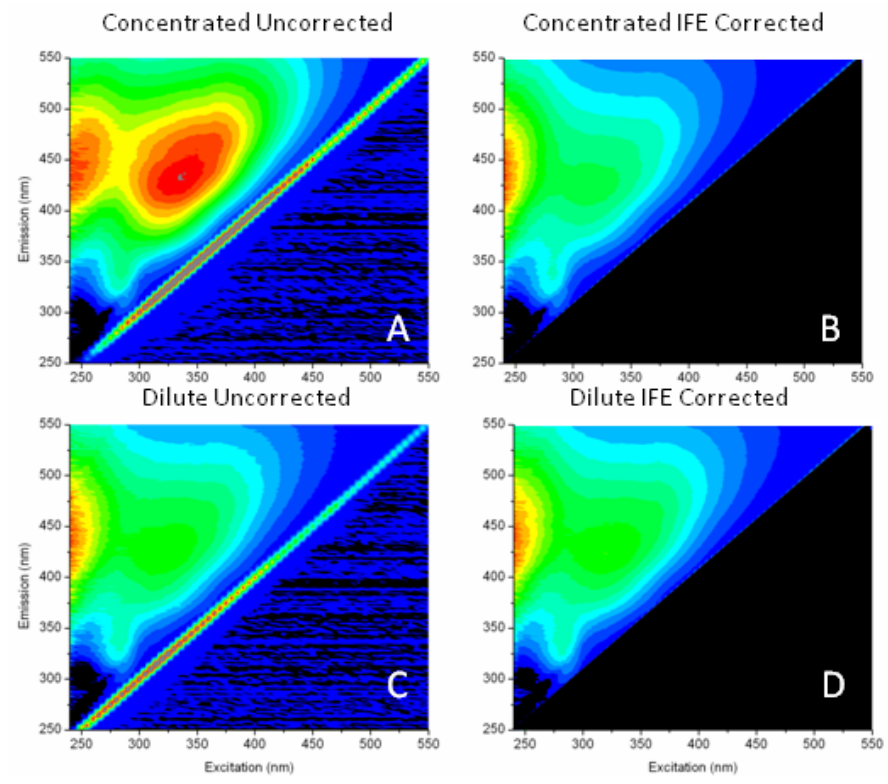

Figure 4: A comparison of the influence of the inner-filter-correction algorithm on EEMs of concentrated (top row) and dilute (bottom row) samples of the Pony Lake Fulvic Acid standard sample. The uncorrected EEMs are shown in panels $\mathrm{A}$ and $\mathrm{C}$; the corrected maps are shown in $\mathrm{B}$ and $\mathrm{D}$, respectively. 

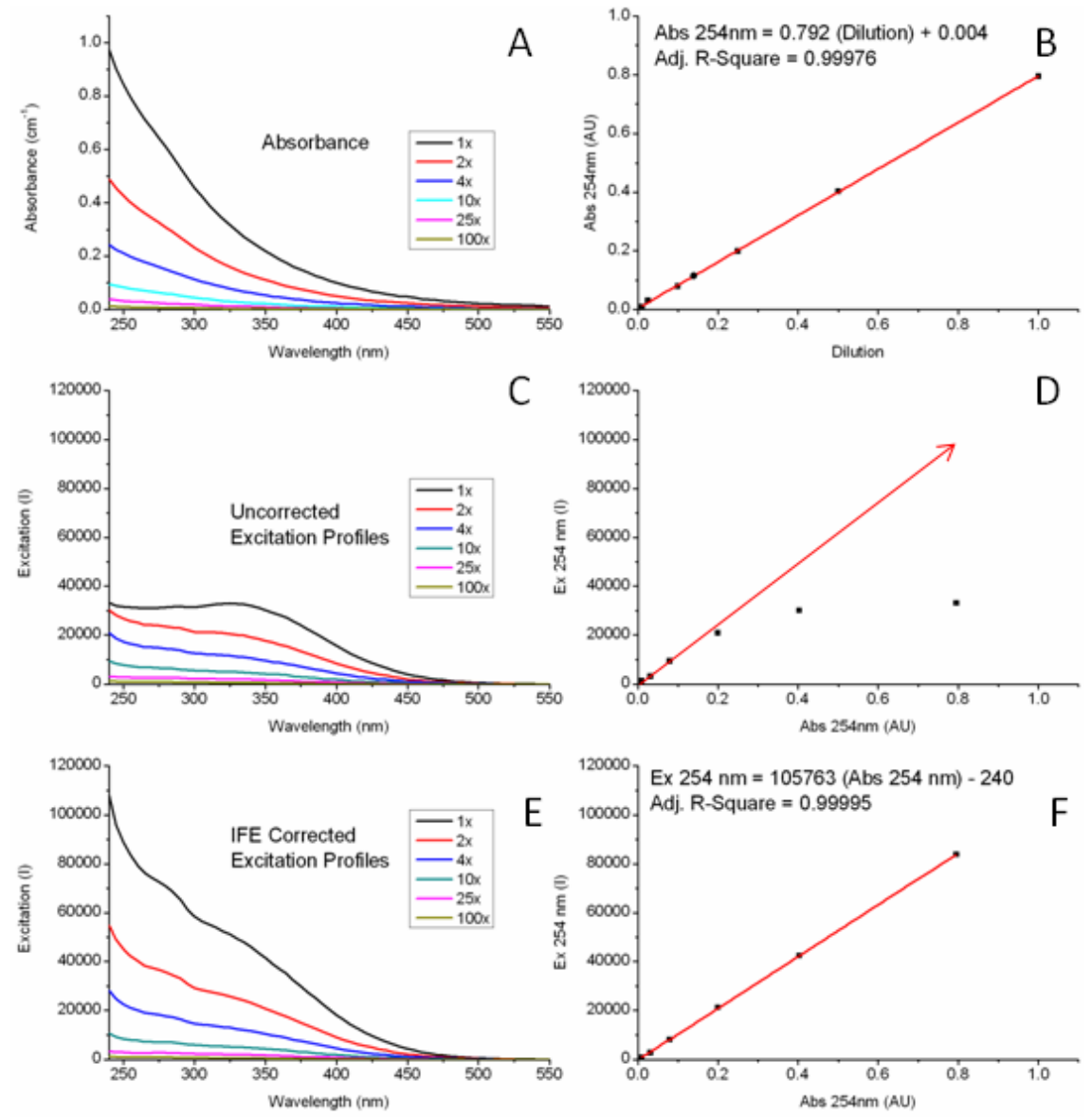

Figure 5: Comparison of the concentration-dependence of the absorbance spectra (A and B) and the excitation spectra of the Pony Lake Fulvic Acid sample before (C and D) and after (E and F) innerfilter correction. The samples were diluted from full strength to 100 -fold as indicated in the legends. Plots of the absorbance versus wavelength are in A; B shows the linear relationship of the absorbance at $254 \mathrm{~nm}$ and the dilution factor. Plots of the excitation spectra representing the integral of the excitation axis are shown in $\mathrm{C}$ versus wavelength. Panel D shows plots of the absorbance value

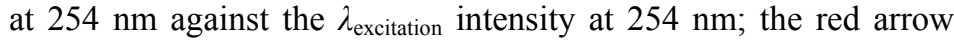
illustrates the nonlinear region when Abs $>0.2$. Panel E shows plots of the excitation spectra from (C) after inner-filter correction, while (D) shows the plot of the linear relationship between the excitation intensity at $254 \mathrm{~nm}$ and the absorbance at $254 \mathrm{~nm}$. 
$>300 \mathrm{~nm}$ on the excitation axis are stronger compared to the contours below 300 $\mathrm{nm}$. This is because the IFE effects are strongest in the UV regions (below 300 $\mathrm{nm})$ where the overlap of component excitation and emission spectra is largest. The IFE correction (fig. 4B) clearly reconstitutes the same contours seen with the dilute sample (fig. 4C) and the IFE correction had little effect on the dilute sample EEM in Figure 4D.

Likewise, Figure 5 shows in more detail the influence of the IFE corrections by comparing the absorbance spectra and the integrated excitation spectra of the PLFA EEM-data samples from a dilution series. Figure 5A shows the absorbance spectra for the PLFA dilution series which are particularly featureless with a quasi-exponential decrease from the UV to visible regions. Figure 5B shows the plot of the absorbance values at $254 \mathrm{~nm}$ from Figure $5 \mathrm{~A}$ versus the dilution factor used in the experiment. The data in Figure $5 \mathrm{~B}$ exhibit a highly significant linear trend up to the peak absorbance near $\mathrm{Abs} \approx 0.8$. Figure $5 \mathrm{C}$ shows that the $F_{\text {obs }}$ for the integrated excitation spectra indicates there is a strong reduction in the intensity below $400 \mathrm{~nm}$. Figure 5D presents no correlation between the absorbance values at $254 \mathrm{~nm}$ ( $x$-axis) and the excitation intensity values at $254 \mathrm{~nm}$ ( $y$-axis) beyond $\mathrm{Abs} \approx 0.2$ due to the increasing IFE. However, as shown in Figure 5E, when the IFE is corrected as described above, the excitation spectral intensity is recovered, closely paralleling the absorbance spectra (Fig. 5A) for the all samples. The highly significant linear relationship between the excitation intensity at $254 \mathrm{~nm}$ and the absorbance at $254 \mathrm{~nm}$ shown in Figure 5F confirms the validity of the IFE correction and its value for concentrated CDOM analysis, allowing comparison to dilute sample spectra.

\section{Spectral analysis and component identification: conventional nonlinear least-squares and multivariate approaches}

As required by the CDOM research community, the concerted application of the instrumental spectral corrections, Rayleigh-line masking, water-Raman subtraction, Raman or QSU normalization and IFE correction are readily enabled by the EEM-processing tools in our AquaLog ${ }^{\circledR}$ software. As mentioned above, the purpose of the spectral corrections and EEM-processing is to make the identification and quantification easier of the CDOM components that are usually based on a reference-component library. Here we focus attention on a popular and promising library-based multivariate technique for CDOM analysis, namely, PARAFAC, which has been documented extensively by researchers including many using HORIBA's fluorescence instruments [1, 3-5]. Importantly the AquaLog ${ }^{\circledR}$ software offers direct access to a MatLab ${ }^{\circledR}$ console for purposes of processing data using the PARAFAC tools in N-way Toolbox, a public-domain package especially developed for CDOM analysis [4]. The modeling advantages of PARAFAC center on its ability to simultaneously evaluate the EEM data as a matrix, and to envelop multiple (often hundreds) of EEMs simultaneously for increased statistical significance [3-5]. PARAFAC has been successful at identifying a wide range of CDOM components including humic and fulvic 
acids, tryptophan- and tyrosine-like substances, quinones, several polycyclic aromatic hydrocarbons, and distinguishing microbial, marine and terrestrial CDOM sources. More importantly, PARAFAC has been used to diagnose trends in CDOM components as a function of several key chemical and physical parameters, including water-recycling-plant treatment stages, sewage dispersion, stream flow, and ocean and estuarial currents, among many others [6]. Indeed, as discussed below, the application of PARAFAC has been proposed as a standard modeling technique for a variety of water-quality applications $[1,14]$.

\section{Conclusion}

Several key publications and international standards documents have recently been published regarding fluorescence-instrument calibration and correction, including EEM data, as well as those focusing on interlaboratory comparisons of CDOM EEM applications. Three recent publications from NIST researchers have particular significance for EEM correction, including a reference to the recently released ASTM standard guide (E2719) for fluorescence calibration and correction [9], a reference to CDOM EEMs and all aspects of instrument and IFE correction, [2] and a reference to the validation of the Fluorolog ${ }^{\circledR}$ as the true accurate fluorometer for its use in generating and validating a series of Standard Reference Materials [7]. Furthermore, a recent paper has been accepted outlining the results of a major international interlaboratory comparison for CDOM IHSS standard samples; this study was headed by researchers and the United States Geological Survey (USGS), and was the focused outcome of a recent 2008 Chapman conference sponsored by the American Geophysical Union [8]. In terms of potential applications of CDOM analysis two reviews of the literature exist of note, including one paper explaining the potential of CDOM for monitoring all stages of water-recycling [14], and another focusing on analysis of natural and wastewater sources [1].

It is clear that fluorescence analysis of CDOM and the use of EEMs will play a central role in water-quality analysis in academic and government research, as well as for various municipal and industrial monitoring applications. HORIBA presently enjoys the recognition of the industry as the leader in both hardware and software for this application. It is our goal to continue to design instrumentation and software and to promote standardization and regulations that will increase the potential for fluorescence as a water-quality analysis tool, and thus help researchers trying to understand, prevent, and treat the consequences of CDOM contamination of our water - one of our most critical resources.

\section{References}

[1] Hudson, N., Baker, A., and Reynolds, D., Fluorescence analysis of dissolved organic matter in natural, waste and polluted waters - A Review, River Research and Applications, 23, pp.631-649, 2007.

[2] Holbrook, R.D., DeRose, P.C., Leigh, S.D., Rukhin, A.L., and Heckert, N.A., Excitation-emission matrix fluorescence spectroscopy for natural 
organic matter characterization: A quantitative evaluation of calibration and spectral correction procedures, Applied Spectroscopy, 60(7), p.791, 2006.

[3] Stedmon, C.A., Markager, S. and Bro, R, Tracing dissolved organic matter in aquatic environments using a new approach to fluorescence spectroscopy, Marine Chemistry, 82, pp.239-254, 2003.

[4] Stedmon, C.A., Markager, S. and Bro. R, Characterizing dissolved organic matter fluorescence with parallel factor analysis: a tutorial, Limnology and Oceanography: Methods, 6, pp.572-579, 2008.

[5] Cory, R.M., and McKnight, D.M. Fluorescence spectroscopy reveals ubiquitous presence of oxidized and reduced quinones in dissolved organic matter, Environmental Science \& Technology, 39,pp.8142-8149, 2005.

[6] Cory, R.M., Miller, M.P., McKnight, D.M., Guerard, J.J., Miller, P.L., Effect of instrument-specific response on the analysis of fulvic acid fluorescence spectra, Limnology and Oceanography: Methods, 8, pp.67-78, 2010.

[7] DeRose, P.C., et al., Qualification of a fluorescence spectrometer for measuring true fluorescence spectra, Review of Scientific Instruments, 78, p.033107, 2007.

[8] Murphy, K.R., Butler, K.D., Spencer, R.G.M., Stedmon, C.A., Boehme, J.R., and Aiken, G.R., The measurement of dissolved organic matter fluorescence in aquatic environments: An interlaboratory comparison, Environmental Science \& Technology, 44(24), pp.9405-9412, 2010.

[9] DeRose, P.C. and Resch-Genger, U., Recommendations for Fluorescence Instrument Qualification: The New ASTM Standard Guide, Analytical Chemistry, 82, pp.2129-2133, 2010.

[10] Gu, Q. and Kenny, J.E., Improvement of Inner Filter Effect Correction Based on Determination of Effective Geometric Parameters Using a Conventional Fluorimeter, Analytical Chemistry, 81, pp.420-426, 2009.

[11] Lakowicz, J. R., Principles of Fluorescence Spectroscopy, $3^{\text {rd }}$ ed., Springer Science and Business Media, LLC: New York, 2006.

[12] Larsson, T., Wedborg, M., and Turner, D., Correction of inner-filter effect in fluorescence excitation-emission matrix spectrometry using Raman scatter. Analytica Chimica Acta, 583, pp.357-363.

[13] MacDonald, B.C., Lvin, S.J., and Patterson, H., Correction of fluorescence inner filter effects and the partitioning of pyrene to dissolved organic carbon, Analytica Chimica Acta, 338, p.155, 1997.

[14] Henderson, R.K., Baker, A., Murphy, K.R., Fluorescence as a potential monitoring tool for recycled water systems: A review, Water Research, 43, p.863, 2010. 